decision-makers (SDMs). SDMs may also be known as 'surrogate' or 'proxy' decision-makers.

Methods A national survey was distributed via an online panel in September 2018 to 1,484 adults aged 18 years and over. Quotas on age, gender and jurisdiction (based on 2017 Australian Census data) aimed to maximise representativeness.

Results Of the 1,058 survey completers (response rate $=71.3 \%$ ), the majority $(67 \%)$ did not know there were laws about substitute decision-making and $12 \%$ had previously made medical decisions on behalf of someone else. Seventyfour percent of those with SDM experience $(n=97)$ agreed that making medical decisions on someone's behalf can be a difficult and stressful experience compared to 56\% of those without SDM experience $(n=589)$. Moreover, only $38 \%$ of those without SDM experience indicated they would feel confident in the role of SDM. When asked their preferred source for receiving SDM information, 59\% of all respondents ranked health professional as their first preference, followed by discussion with family or friends (23\%), traditional media (7\%), new media (6\%) or an event (3\%). Only $16 \%$ indicated that appointing a SDM was a priority at the time of completing the survey.

Conclusion(s) Among a representative sample of Australians it was relatively common to have acted in the role of SDM and most who had perceived the role as challenging. Further education and support is needed to clarify roles, relevance and benefits in appointing and preparing SDMs.

\section{OP53 A SYSTEMATIC REVIEW OF ECONOMIC EVALUATIONS OF ADVANCE CARE PLANNING: DATA LIMITATIONS AND ETHICAL CONSIDERATIONS}

M Nesari* ${ }^{*}$ M Douglas, S Ghosh, P Biondo, N Hagen, J Simon, K Fassbender. University of Alberta, Edmonton, Canada

\subsection{6/spcare-2019-ACPICONGRESSABS.53}

Background Evidence regarding the degree and direction of economic impacts of implementing Advance Care Planning (ACP) is inconsistent. Also, available reviews have not systematically assessed the quality of the costing data in the primary studies. We aimed to synthesize current evidence on the economic impacts of implementing ACP and explore implications for policy and practice.

Methods We conducted a comprehensive search of online bibliographic databases. Reference lists of included articles were also reviewed. We assessed the quality of costing in studies using the Consensus on Health Economics Criteria Checklist (CHEC).

Results We included 33 studies; the majority were from the USA $(78.8 \%)$. Studies were conducted in various settings, mostly hospitals (60\%). Almost $64 \%$ of studies reported cost savings from the healthcare systems' perspectives; no study included patients' perspectives (out-of-pocket-costs). Assessing quality of costing using CHEC revealed weaknesses in studies including: flaws with costs identification (37.9\%), measurement $(39.3 \%)$, and valuation $(44.8 \%)$; no consideration of intervention costs $(87.9 \%)$; not including all relevant variables in sensitivity analyses (34.5\%); and not discounting the costs $(55.6 \%)$.
Discussion We detected substantial methodological issues with current economic evaluations of ACP that compromise the validity of evidence. To inform policy makers about ACP, which is a multifaceted process, methodologically robust studies are needed that capture costs of the program from all major payers. A comprehensive report on cost evaluations is highly recommended. Meanwhile, respecting patient choice remains a valid clinical basis for promoting use of ACP.

\section{OP54 DELIVERING SYSTEM-WIDE ADVANCE CARE PLANNING SUPPORT IN REAL-WORLD SETTINGS: ECONOMIC CONSIDERATIONS. AN EXPLORATORY, QUALITATIVE STUDY IN TWELVE INTERNATIONAL HEALTHCARE ORGANISATIONS}

J Dixon*, M Knapp. London School of Economics and Political Science, London, UK

\subsection{6/spcare-2019-ACPICONGRESSABS.54}

Background Facilitation of ACP conversations is time consuming, whether undertaken in one or multiple shorter discussions. Our exploratory, qualitative study in twelve healthcare systems (US, Canada, New Zealand, Australia) providing system-wide ACP support explored:

- organizational rationales for provision, including perspectives on the economic case

- type and organization of staffing

- ways of providing high-quality, system-wide support costefficiently.

Methods Interviews with leaders, ACP specialists, physicians, nurses, social workers and others (average $n=13$ ) were conducted in twelve purposively-sampled healthcare systems. Data were transcribed and thematically analysed using NVivo software.

Results System-wide ACP support was primarily a strategic response to risks associated with increased availability and use of life-prolonging interventions in serious illness and frailty. Overall cost-savings were not expected.

Staffing ACP support was challenging. While professionals often needed more protected time, promising approaches included team-based provision, especially physicians working with nurses and social workers, and systematic incorporation into chronic and routine care.

Skilled and experienced staff underpinned cost-effective provision. While dedicated facilitators were not scalable or sustainable, some level of specialism and voluntarism, with plentiful opportunities to develop skills in practice, was indicated.

ACP support was provided equally efficiently by experienced staff regardless of guides or approach used. Serious illness conversations could build on earlier ACP support. Community- and group-based approaches were thought costefficient, increasing reach and supporting later planning and decision-making.

Conclusions Investments in ACP support were justified by management of organizational risk and high-quality patient care. Our findings identify areas where cost-efficiencies in provision of system-wide ACP support may be found 